\title{
Effects of Hip Arthroplasties on Bone Adaptation in Lower Limbs: A Computational Study
}

\author{
Abdul Halim Abdullah ${ }^{1,2}$, Mitsugu Todo 3 \\ ${ }^{1}$ Interdisciplinary Graduate School of Engineering and Sciences, Kyushu University, Fukuoka, Japan \\ ${ }^{2}$ Fakulti Kejuruteraan Mekanikal, Universiti Teknologi MARA, Shah Alam, Malaysia \\ ${ }^{3}$ Research Institute for Applied Mechanics, Kyushu University, Fukuoka, Japan \\ Email: abdul.halim.abdullah.822@s.kyushu-u.ac.jp, todo@riam.kyushu.ac.jp
}

Received December 2014

\section{Abstract}

Gait disorders contribute to the risk of falls and successive injuries, especially to elderly populations. The risk of falls becomes higher for hip osteoarthritis $(\mathrm{OA})$ and hip arthroplasties patients due to poor balancing and gait impairment. Bone adaptation and bone loss are fundamental issues in considering the changes of bone behavior and gait pattern. In this study, computational analysis of the lower limbs was conducted to estimate the bone adaptation after hip arthroplasties procedure. 3D inhomogeneous model of lower limb was developed from computed topography (CTbased) data of 79 years old patient with hip osteoarthritis problem in left limb. Two types of arthroplaties were constructed in the left limb, namely total hip arthroplasty and resurfacing hip arthroplasty using commercial biomedical software, Mechanical Finder v6.1. Prosthesis stem and acetabular cup of THA were modelled as titanium alloy material $(E=114 \mathrm{GPa}, v=0.34)$, femoral ball and bearing insert as alumina properties $(E=370 \mathrm{GPa}, v=0.22)$. Meanwhile, RHA implant was assigned as Co-Cr-Mo material $(E=230 \mathrm{GPa}, v=0.30)$. Contact between both implants and bone were considered to be perfectly bonded at the interface. A load case of quiet standing position was conducted in this analysis with $60 \mathrm{~kg}$ of the patients' body weight. The load was applied at the cross sectional lumbar vertebra and fixed at the distal of femoral shafts. Results show different patterns of stress distribution in right and left (operated) limbs for hip OA, THA and RHA models. An indication of stress alteration on both limbs after arthroplasties suggested that the bone adaptation occurred. The higher percentage of change in the left limb projected that the adaptation was more critical in operated limb.

\section{Keywords}

Lower Limbs, Osteoarthritis, Total Hip Arthroplasty, Resurfacing Hip, Bone Adaptation

\section{Introduction}

Total hip arthroplasty (THA) and resurfacing hip arthroplasty (RHA) are two approaches that commonly used 
for hip osteoarthritis (OA) treatment. Advantage and disadvantage of each procedure were still discussed till nowadays, especially in promoting long term stability. In general, RHA approaches only requires a small amount of femoral head removal or resurfacing techniques while THA demand for total replacement. Continuous improvement of surgical techniques and design of implant [1] [2] had encouraged the long term performance. Concern of bone remodeling, prosthetic loosening [3] and gait performance after surgery [4] are a few criteria that mostly relate to the survival of the arthroplasty process. Above all, understanding the bone adaptation factor plays an important role in considering the performance at primary stage. Changes of bone behavior were initially predicted on the resulting stress and strain distribution.

Computational analysis had extensively used to predict the stability and performance of hip arthroplasties. Most studies were focused on the affected (osteoarthritis) and operated (arthroplasties) limb where the prosthesis implanted. Unfortunately, the hip OA disease presence had altered the performance of both affected and nonaffected limbs [5]. The similar observation applied with the presence of artificial hip joint which also reflects to non-operated limb [6] [7]. In rehabilitation study, Kiss (2010) [5] had recognized that the motion and functional abilities of affected hip was restricted while non-affected hip demonstrate a compensation mechanism for gait stability [8]. Correspondingly, the non-operated limb was expected to demonstrate greater force and moment to compensate for the operated limb. Therefore, the development of lower limb model in this study was important to predict weight distribution and bone adaptation in both operated and non-operated limbs.

The objective of this paper was to investigate the effects of different hip arthroplasties on bone adaptation in lower limb, namely total hip arthroplasty (THA) and resurfacing hip arthroplasty (RHA). Finite element analysis on both operated and non-operated limbs was conducted to promote primary understanding of gait stability.

\section{Material \& Methods}

\subsection{Development of Finite Element Model}

Finite element (FE) model of the lower limb employed in this analysis was constructed based on computed tomography (CT) image data of a 79-year old female patient with hip osteoarthritis (OA), collected from Kyushu University Hospital, Japan. Different gray scales extracted from the CT image presenting the variety of bone density and consequently creating the inhomogeneous 3D model. The procedure was conducted using commercial biomedical software, Mechanical Finder v7.0 while estimation of young modulus distribution was generated based on Keyak et al. [9]. Description of the calculation was indicated in Table 1 while FE model and variant of young modulus was illustrated in Figure 1.

\subsection{Material Properties of Hip Arthroplasties}

Two types of arthroplasties were considered in this study, namely total hip arthroplasty (THA) and resurfacing hip arthroplasty (RHA). Both were modeled based on commercial products and implanted into the left limb which was suffering from hip osteoarthritis (OA). The hip cartilage model was defined as homogeneous in the lower limb model with elastic modulus of $0.004 \mathrm{GPa}$ and Poisson ratio of 0.4. In THA model, ceramic-on-ceramic (CoC) type of implant was used which presenting Alumina ceramic as femoral ball and acetabular liner. The prosthesis stem and acetabular cup were assigned as biocompatibility material, namely titanium alloy. Meanwhile, cobalt chrome material was determined in the RHA model for both acetabular cup and prosthesis pin. Illustration of lower limbs with THA and RHA was shown in Figure 2 while the details of material properties used in both arthroplasties were described in Table 2.

Table 1. Estimation for young modulus and yield strength of inhomogeneous bone [4].

\begin{tabular}{|c|c|c|c|}
\hline Density Range & Young Modulus (MPa) & Density Range & Yield Strength (MPa) \\
\hline$\rho=0$ & $E=0.001$ & & \\
\hline $0<\rho \leq 0.27$ & $\mathrm{E}=33900 \rho^{2.20}$ & $\rho \leq 0.2$ & $\sigma_{\text {yield }}=1.0 \times 10^{20}$ \\
\hline $0.27<\rho<0.6$ & $\mathrm{E}=5307 \rho+469$ & $0.2<\rho<0.317$ & $\sigma_{\text {yield }}=137 \rho^{1.88}$ \\
\hline $0.6 \leq \rho$ & $\mathrm{E}=10200 \rho^{2.01}$ & $0.317 \leq \rho$ & $\sigma_{\text {yield }}=114 \rho^{1.72}$ \\
\hline$\rho=0$ & $E=0.001$ & & \\
\hline
\end{tabular}


Table 2. Mechanical properties of total hip and resurfacing hip arthroplasties.

\begin{tabular}{|c|c|c|c|c|}
\hline & Model & Material & Elastic Modulus, E (GPa) & Poisson Ratio, v \\
\hline \multirow{4}{*}{ THA } & Acetabular cup & Titanium Alloy & 114 & 0.34 \\
\hline & Bearing liner & Alumina & 370 & 0.23 \\
\hline & Femoral ball & Alumina & 370 & 0.23 \\
\hline & Prosthesis stem & Titanium Alloy & 114 & 0.34 \\
\hline \multirow{2}{*}{ RHA } & Acetabular cup & Cobalt Chrome & 230 & 0.30 \\
\hline & Prosthesis pin & Cobalt Chrome & 230 & 0.30 \\
\hline
\end{tabular}

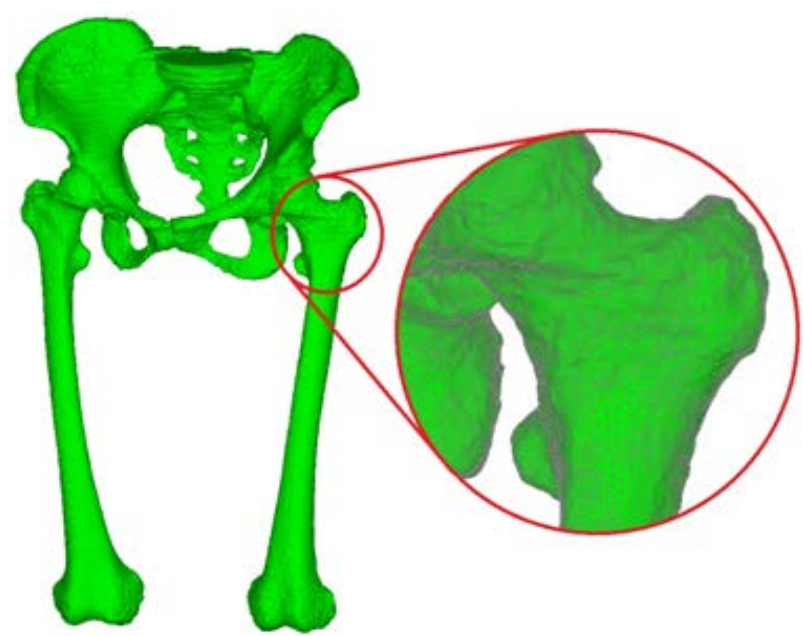

(a)

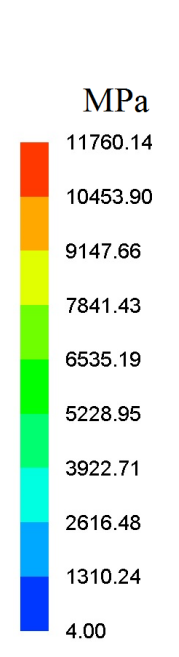

00

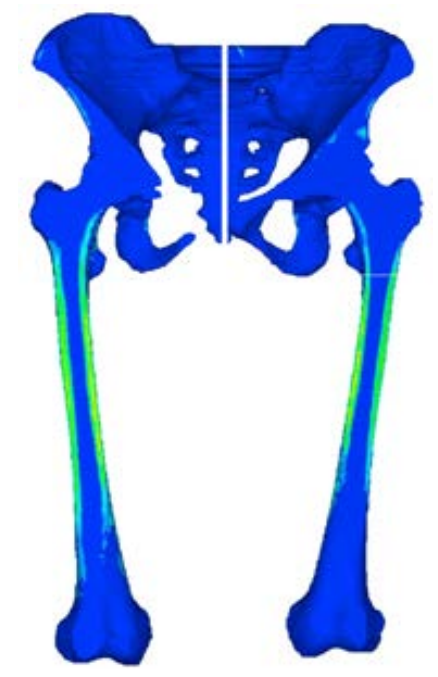

(b)

Figure 1. (a) Finite element model of lower limbs and (b) Distribution of young modulus in inhomogeneous model.

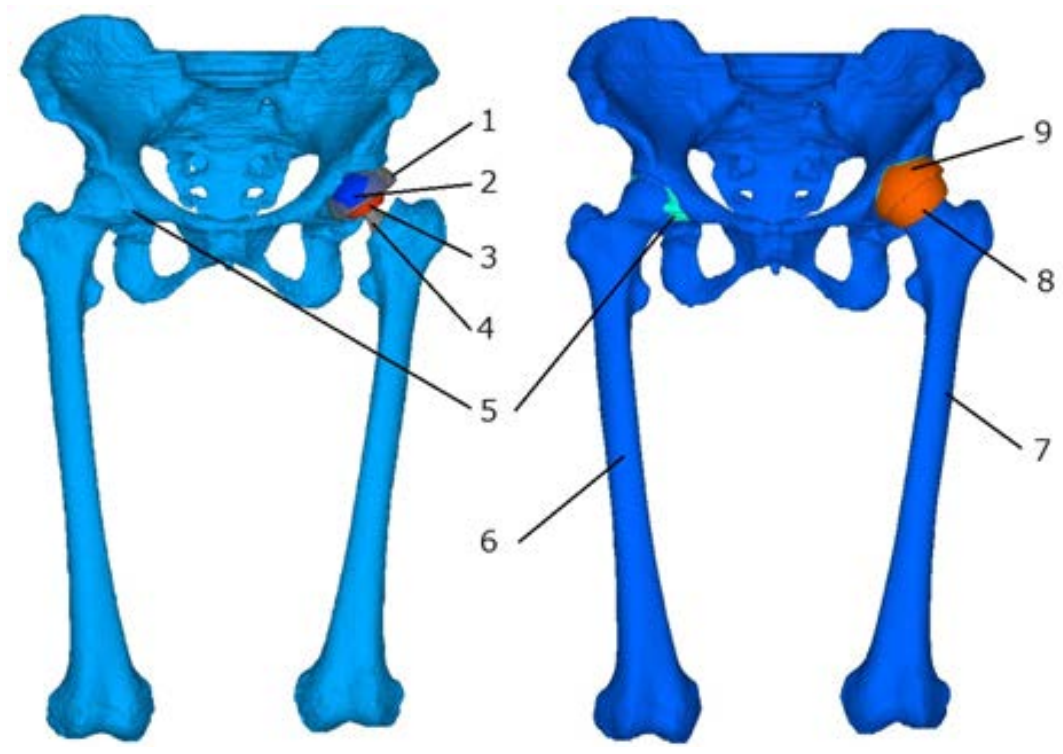

(a)

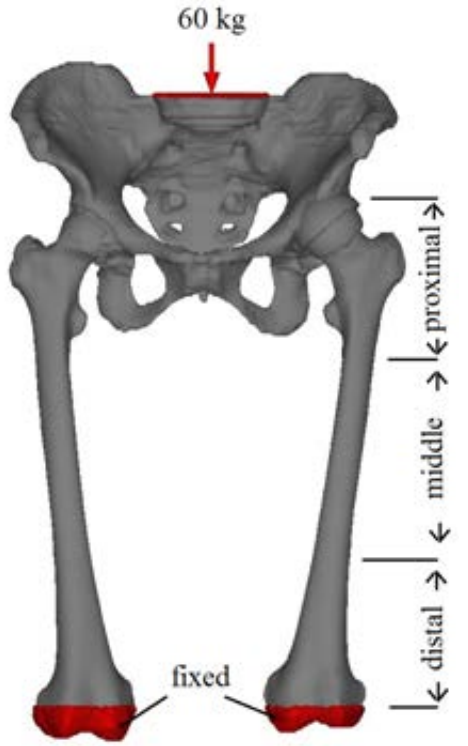

(b)

Figure 2. (a) 3D model of lower limbs with THA (left) and RHA (right) on left limb. (1) Acetabular cup; (2) Bearing liner; (3) Femoral head; (4) Prosthesis stem; (5) Hip cartilage; (6) Right limb; (7) Left limb; (8) Prosthesis pin and (9) Acetabular cup. (b) Description of loading, boundary condition and designated regions. 


\subsection{Loading \& Boundary Conditions}

The posture of quite standing with foot side-by-side position contributes to functional and structural equivalent in the lower limbs [10]. Thus, the respective loading condition was considered in this study by applying the patients' body weight of $60 \mathrm{~kg}$ to the cross-sectional of a lumbar vertebra. The distal ends of the femoral shaft were fixed at all directions for boundary condition. All three models were analyzed on the similar loading condition to predict the bone adaptation effects in the lower limbs with hip arthroplasties. The description of the loading and boundary condition was illustrated in Figure 2(b).

\section{Results \& Discussion}

Biomechanical behavior and prediction of bone adaptation in the analysis was presented in corresponding to equivalent stress (Drucker-Prager) distribution and changes of maximum principal stress after implantation. The observation of the stress alteration was conducted on both operated and non-operated limbs which further estimated the contribution to gait adaptation.

\subsection{Distribution of Equivalent Stress in Lower Limbs with Arthroplasties}

Stress shielding phenomenon is a common issue that related to hip arthroplasties. It occurred when there was a mismatch in the elastic modulus of two materials. The presence of stiffer materials of arthroplasties will dominate the bodyweight loading and consequently unloaded the surrounding bones. Bones with reduced load were expected to experience bone resorption and become weak [11]. Results shown in Figures 3(a)-(c) indicated the variation of equivalent stress in three different models of lower limbs which representing limb with hip OA, THA and THA, respectively. A similar scale was applied in all cases to ease assessment and comparison. Different stress variations were predicted in lower limbs after arthroplasties especially in the left (operated) limb. In hip OA model, pattern of stress variation in left and right limbs were different. On left limb which were affected by hip OA, higher stress was predicted on femoral shaft. Stress was concentrated at the femoral neck and the distal end of the femoral shaft in right limb. A different variation on both limbs may caused by osteoarthritis effects on left hip joint. Biomechanically, hip OA experienced lesser elastic modulus as compared to healthy bone. Besides, alteration of the physical structure and shape due to the disease may contribute to the findings [12].

In THA lower limb model as shown in Figure 3(b), the pattern of stress distribution was obviously differed at left limb. Proximal region of femoral shaft experienced lesser stress while higher stress at middle and distal regions. Meanwhile, only small changes were estimated in the right limb as compared to hip OA model. The existence of stiffer prosthesis stem and the procedure of femoral head removal in THA contributed to the reduced stress in the bone. Dissimilar findings in RHA lower limb, stress was decreased in all regions of femoral shaft

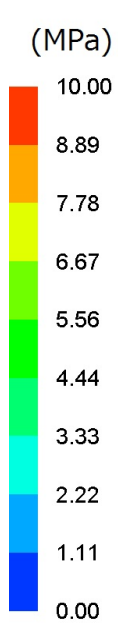

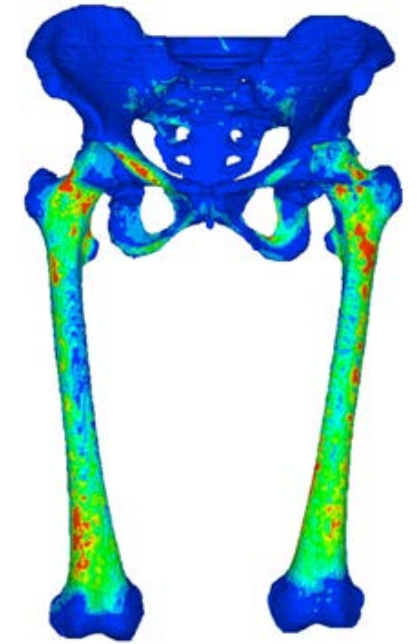

(a)

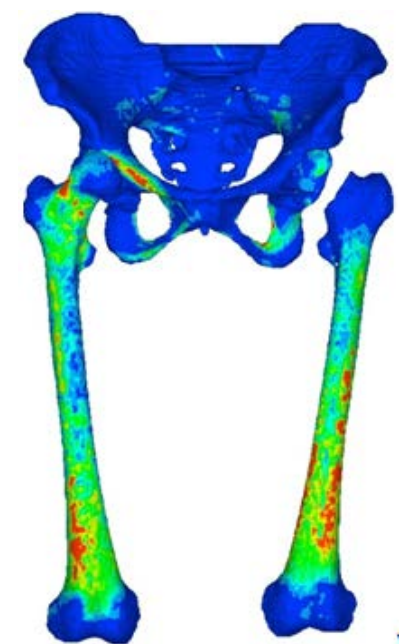

(b)

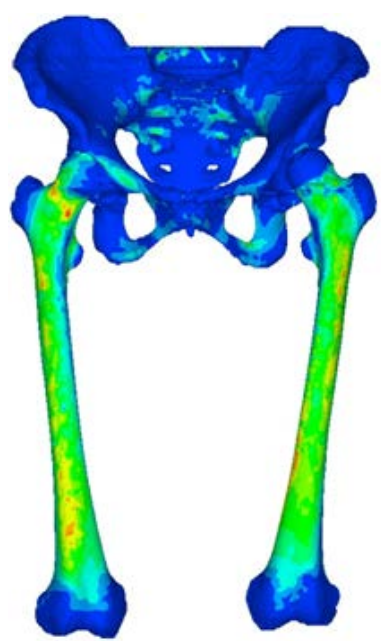

(c)

Figure 3. Variation of equivalent stress (Drucker-Prager) in lower limbs with (a) hip OA, (b) THA and (c) RHA. 
after the implantation. The right limb also experienced similar findings, but in smaller scale. However, the pattern of stress almost similar to that observed in hip OA model. The results were significant as the implant in RHA only required for minimal bone removal and the implant was designed as cobalt chrome material with a higher modulus of elasticity.

\subsection{Prediction of Bone Adaptation in Operated and Non-Operated Limb}

Three different regions in femoral shaft were assigned to observed stress behavior which were proximal, middle and distal region. The peak value of maximum principal stress of the respective region was selected to calculate the prediction of stress and bone adaptation after arthroplasties. Figure 4 illustrated the cross-sectional view of operating limb after THA and comparison of the peak value of maximum principal stress at the respective regions. Results showed that in proximal region, both operated limbs experienced reduced stress which might lead to bone resorption. Limb with THA indicated 46.25 percent of stress reduced while RHA limb at 53.71 percent. Presence of prosthesis stem in lower limbs had created a mismatch material in the bone, which lead to stress shielding phenomena and further contributes to biomechanical instability. At medial and distal regions, increased stress was measured in THA model up to 58.34 and 39.08 percent, respectively. The bone thickening was predicted as the stress was totally transferred to the bone at the distal end of prosthesis stem. Meanwhile, the whole operated limb with RHA were projected to experience bone resorption as middle and distal regions also indicated reduced stress around 20 - 45 percent.

Performance of non-operated limb was normally neglected in analysis as most researchers more interested and concentrated in operating limb. However, research of rehabilitation studies had observed that the gait adaptation was also occurring in non-operated limb of arthroplasties' patient [13]. In biomechanical point of views, the problems might be initiated by the changes of bone behavior after arthroplasty procedure. Thus, evaluation and analysis of the non-operated limb might contribute to the understanding of bone and gait adaptation. Findings of non-operated limb as illustrated in Figure $\mathbf{5}$ showed that stress was reduced at all regions for both THA and RHA cases. The comparison was made between the arthroplasty model and hip OA model to estimate the bone adaptation. Right limb for RHA model experienced more stress changes as compare to THA model. The alteration was calculated up to 40.26 percent, 29.21 percent and 38.2 percent at proximal, middle and distal region, respectively. Meanwhile, only minimum changes were predicted in THA model around 6 to 18 percent.

The factor of stiffer material of implant might be the reason of great differences (reduced magnitude) in RHA model. However, the pattern of stress distribution in the model was observed to be almost similar to that predicted in hip OA model. Instead of smaller changes, proper stress distribution along the limb was also important as it will promote bone growth and long term stability [14]. The current findings were supported by a rehabil-

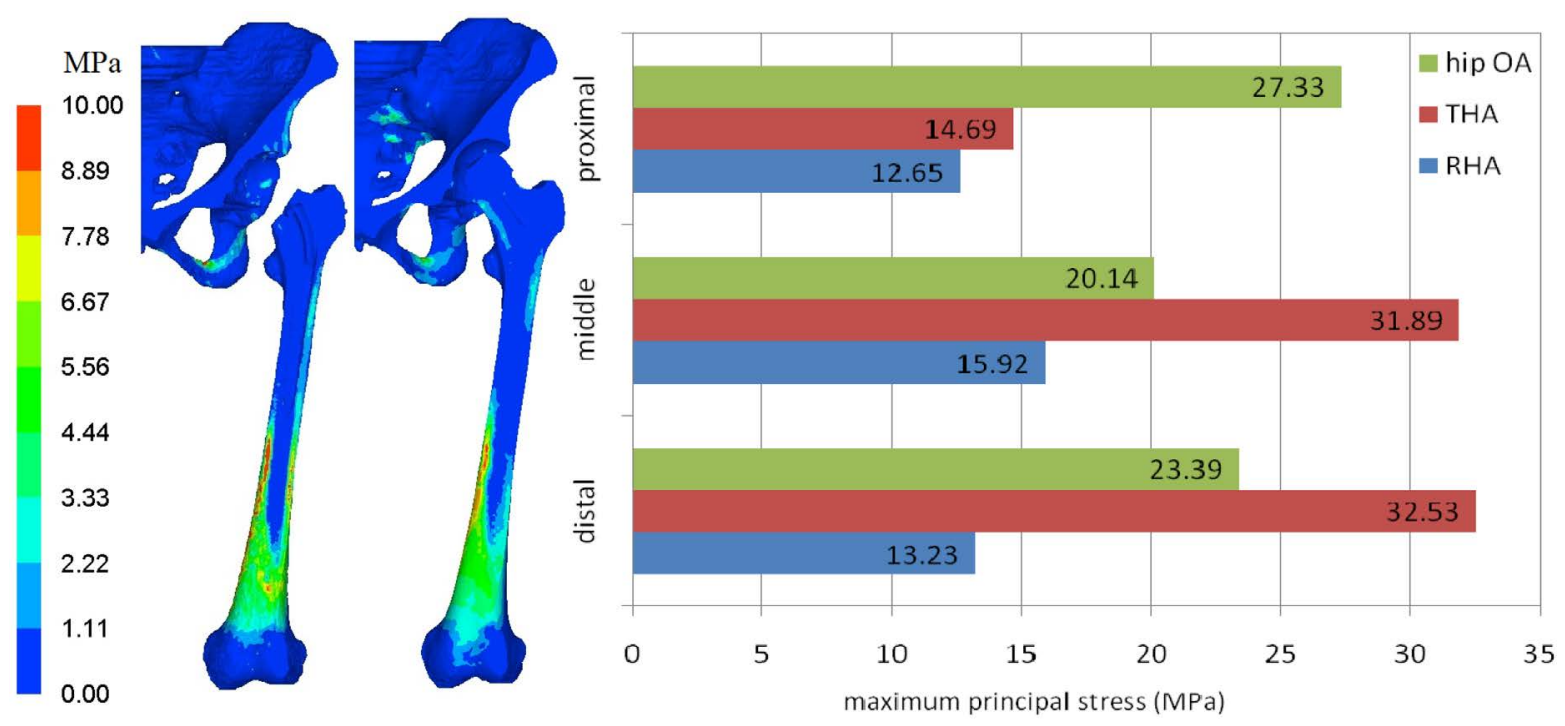

Figure 4. Cross-sectional view operated limbs and the peak value of maximum principal stress in a left limb model at different regions to estimate bone adaptation. 

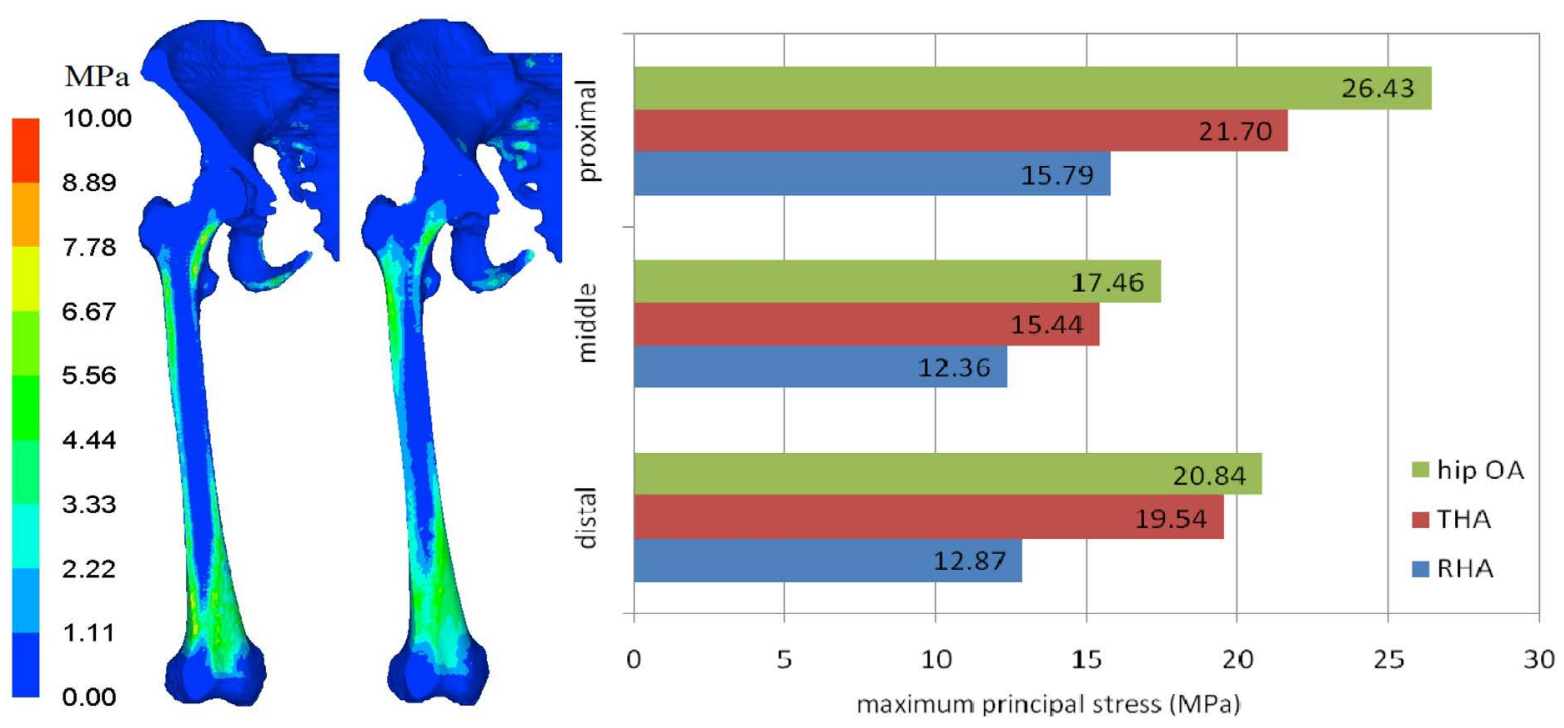

Figure 5. Cross-sectional view non-operated limbs and the peak value of maximum principal stress in right limb for THA (left) and RHA (right) models at different regions.

itation study, which reported the characteristic of surface hip arthroplasty allowed the gait to return to most normative pattern compared with THA [4].

A number of limitations in the present study were identified. Variability of different physiological loading and consideration of muscle reaction was not conducted. Body-mass index (BMI) and muscle strength factors were contributed to gait balancing and stability [15] [16], further evaluation of stress-strain stimuli and muscle reaction in lower limbs should be considered for better estimation.

\section{Conclusion}

The results of the present study discovered basic understanding of load distribution in the lower limb and prediction of bone adaptation after arthroplasty on the resulting stress distribution. Changes of stress variation in both limbs suggested both operated and non-operated limb were potential to experience bone adaptation. Lower limbs with resurfacing hip arthroplasty indicated a similar pattern of stress distribution to that predicted in hip osteoarthritis model. Stress alteration was more critical in RHA limb compared with THA limb model.

\section{Acknowledgements}

The authors would like to acknowledge with gratitude Prof. Yukihide Iwamoto and Assoc. Prof Yasuharu Nakashima from Kyushu University Hospital for their assistance in providing CT scan data from the hospital. This research was supported, in part, by Ministry of Education Malaysia.

\section{References}

[1] Marker, D.R., Strimbu, K., McGrath, M.S., Zywiel, M.G. and Mont, M.A. (2009) Resurfacing versus Conventional Total Hip Arthroplasty: Review of Comparative Clinical and Basic Science Studies. Bulletin of the NYU Hospital for Joint Diseases, 67, 120-127.

[2] Joshi, M., Advani, S., Miller, F. and Santare, M. (2000) Analysis of a Femoral Hip Prosthesis Designed to Reduce Stress. Journal of Biomechanics, 33, 1655-1662. http://dx.doi.org/10.1016/S0021-9290(00)00110-X

[3] Boyle, C. and Kim, I.Y. (2011) Comparison of Different Hip Prosthesis Shapes Considering Micro-Level Bone Remodeling and Stress-Shielding Criteria Using Three-Dimensional Design Space Topology Optimization. Journal of Biomechanics, 44, 1722-1728. http://dx.doi.org/10.1016/j.jbiomech.2010.11.029

[4] Nantel, J., Termoz, N., Vendittoli, P.A., Lavigne, M. and Prince, F. (2009) Gait Patterns after Total Hip Arthroplasty and Surface Replacement Arthroplasty. Arch Phys Med Rehabil, 90, 463-469.

http://dx.doi.org/10.1016/j.apmr.2008.08.215 
[5] Kiss, R.M. (2010) Effects of Walking Speed and Severity of Hip Osteoarthritis on Gait Variability. Journal of Electromyography and Kinesiology, 20, 1044-1051. http://dx.doi.org/10.1016/j.jelekin.2010.08.005

[6] Holnapy, G., Iiiyes, A. and Kiss, R.M. (2013) Impact of the Method of Exposure in Total Hip Arthroplasty on the Variability of Gait in the First 6 Months of the Postoperative Period. Journal of Electromyography and Kinesiology, 23, 966-976. http://dx.doi.org/10.1016/j.jelekin.2013.01.005

[7] Bennet, D., Ogonda, L., Ellitt, D., Humphreys, L., Lawlor, M. and Beverland, D. (2007) Comparison of Immediate Postoperative Walking Ability in Patients Receiving Minimally Invasive and Standard-Incision Hip Arthroplasty. Journal of Arthroplasty, 22, 490-495. http://dx.doi.org/10.1016/j.arth.2006.02.173

[8] Dujardin, F., Aucouturier, T., Bocquet, G., Dupare, F., Weber, J. and Thormine, J.M. (1998) Kinematics of the Healthy and Arthritic Hip Joint during Walking. A Study of 136 Subjects. Rev Chir Orthop Reparatrice Appar Mot, 84, 689699.

[9] Keyak, J.H., Skinner, H.B. and Fleming, J.A. (2001) Effect of Force Direction on Femoral Fracture Load for Two Types of Loading Conditions. J Orthop Res, 19, 539-544. http://dx.doi.org/10.1016/S0736-0266(00)00046-2

[10] Recnik, G., Kralj-Iglic, V., Iglic, A., Antolic, V., Kramberger, S. and Vengust, R. (2007) Higher Peak Contact Hip Stress Predetermines the Side of Hip Involved in Idiopathic Osteoarthritis. Clinical Biomechanics, 22, 1119-1124. http://dx.doi.org/10.1016/j.clinbiomech.2007.08.002

[11] Van Rietbergen, B., Huiskes, R., Weinans, H., Sumner, D.R., Turner, T.M. and Galante, J.O. (1993) The Mechanism of Bone Remodeling and Resorption around Press-Fitted THA Stems. Journal of Biomechanics, 26, 369-382. http://dx.doi.org/10.1016/0021-9290(93)90001-U

[12] Teichtahl, A.J., Wang, Y., Smith, S., Wluka, A.E., Giles, G.G., Bennell, K.L., O’Sullivan, R. and Cicuttini, F.M. (2014) Structural Changes of Hip Osteoarthritis using Magnetic Resonance Imaging. Arthritis Research \& Therapy, 16, 466475. http://dx.doi.org/10.1186/s13075-014-0466-4

[13] Beaulieu, M.L., Lamontagne, M. and Beaule, P.E. (2010) Lower Limb Biomechanics during Gait Do Not Return to Normal Following Total Hip Arthroplasty. Gait and Posture, 32, 269-273. http://dx.doi.org/10.1016/j.gaitpost.2010.05.007

[14] Abdullah, A.H., Jaafar, E.F., Nasir, N., Abdul Latip, E.N. and Tardan, G. (2011) Influences of Prosthesis Stem Lengths in Cementless Total Hip Arthroplasty. Applied Mechanics and Materials, 52-54, 2088-2093. http://dx.doi.org/10.4028/www.scientific.net/AMM.52-54.2088

[15] Chung, C.Y., Park, M.S., Lee, K.M., Lee, S.H., Kim, T.K., Kim, K.W., Park, J.H. and Lee, J.J. (2010) Hip Osteoarthritis and Risk Factors in Elderly Korean Population. Osteoarthritis and Cartilage, 18, 312-316. http://dx.doi.org/10.1016/j.joca.2009.11.004

[16] Ringsberg, K., Gerdhem, P., Johansson, J. and Obrant, K.J. (1999) Is There a Relationship between Balance, Gait Performance and Muscular Strength in 75-Year-Old Women? Age and Aging, 28, 289-293. http://dx.doi.org/10.1093/ageing/28.3.289 